\title{
Dynamics of a Grain-Filled Ball on a Vibrating Plate
}

\author{
F. Pacheco-Vázquez, ${ }^{1, *}$ F. Ludewig, ${ }^{2}$ and S. Dorbolo ${ }^{2}$ \\ ${ }^{1}$ Instituto de Física, Benemérita Universidad Autónoma de Puebla, Apartado Postal J-48, Puebla 72570, Mexico \\ ${ }^{2}$ GRASP, Physics Department B5, Université de Liège, B4000-Liège, Belgium
}

(Received 12 February 2014; revised manuscript received 6 June 2014; published 10 September 2014)

\begin{abstract}
We study experimentally how the bouncing dynamics of a hollow ball on a vibrating plate is modified when it is partially filled with liquid or grains. Whereas empty and liquid-filled balls display a dominant chaotic dynamics, a ball with grains exhibits a rich variety of stationary states, determined by the grain size and filling volume. In the collisional regime, i.e., when the energy injected to the system is mainly dissipated by interparticle collisions, an unexpected period- 1 orbit appears independently of the vibration conditions, over a wide range. This is a self-regulated state driven by the formation and collapse of a granular gas within the ball during one cycle. In the frictional regime (dissipation dominated by friction), the grains move collectively and generate different patterns and steady modes: oscillons, waves, period doubling, etc. From a phase diagram and a geometrical analysis, we deduce that these modes are the result of a coupling (synchronization) between the vibrating plate frequency and the trajectory followed by the particles inside the cavity.
\end{abstract}

DOI: 10.1103/PhysRevLett.113.118001

PACS numbers: 45.70.-n, 45.50.Tn, 45.70.Qj

A bouncing ball (BB) on a vibrated plate is a fundamental system used as a model in different physical and engineering problems [1]; for instance, it has been used to describe cosmic-ray particles in astrophysics [2], the dynamic stability in human performance [3], and the cantilever motion in atomic force microscopy [4]. The BB dynamics displays a period doubling route to chaos also found in biological, hydrodynamic, optical, and chemical systems [5]. More complex objects such as dimers [6], trimers [7], bouncing droplets [8], and quantum bouncers [9] also show nonlinear behaviors: bifurcations, rotations, etc. Dimers and trimers exhibit self-propulsion, providing models to study collective motions, such as fish schools [10], flocks [11], and bacteria colonies [12]. Clearly, the study of bouncing objects has been relevant to the description of a large variety of nonlinear behaviors.

This Letter explores the bouncing dynamics of a hollow sphere partially filled with grains. We aim to understand how the classical BB dynamics (widely studied in the literature $[1,5,13,14])$ is affected by the fast energy dissipation typical of granular materials. We compared the dynamics of an empty, a liquid-filled, and a grain-filled sphere. The former two cases exhibit chaotic behaviors while the sphere with grains bounces primarily in steady states. Of particular interest was the observation of a period-1 orbit independent of the vibration conditions in a broad range. This state was observed at low filling ratios $V_{f}(<12 \%)$ and using large beads (diameter $d=2 \mathrm{~mm}$ ). When tiny particles were used $(300 \mu \mathrm{m})$, different patterns appeared depending on the vibration frequency $f$, for instance, stable oscillons and surface waves similar to those observed in vibrated granular beds [15-19]. The above grain size dependence reveals, as in the case of granular dampers [20-25], the relevance of the main dissipation mechanism: interparticle collisions for large particles and friction as the particle size decreases.

Experimental setup.-A transparent ping-pong ball (coefficient of restitution $\epsilon_{c} \approx 0.91 \pm 0.02$, mass $m_{c}=$ $1.9 \pm 0.1 \mathrm{~g}$, and inner or outer diameter $D_{c}=3.75 / 3.80 \pm$ $0.02 \mathrm{~cm}$ ) was partially filled with liquid or grains and placed on a steel plate (mass $=240 \mathrm{~g}$ ) subjected to vertical oscillations $S(t)=A \sin (\omega t)$; here, $A$ is the vibration amplitude and $\omega=2 \pi f$. To ensure that the ball was bouncing, we used a dimensionless acceleration $\Gamma=A \omega^{2} / g>1$. We used a vibration system TV50101/LS-80 (moving element mass $=1.6 \mathrm{~kg}$ ) and a LABVIEW program to automatically set $\Gamma$ and reduce it from $\Gamma_{\max }=10$ to $\Gamma_{\min }=1$ (at fixed $f$ ) by constant steps $\Delta \Gamma=0.025$ every $25 \mathrm{~s}$. The time of flight between two consecutive collisions $\Delta t$ was measured using an electrical circuit as sketched in Fig. 1: a thin aluminum foil carefully glued at the external bottom of the sphere was soldered to a copper wire of negligible mass

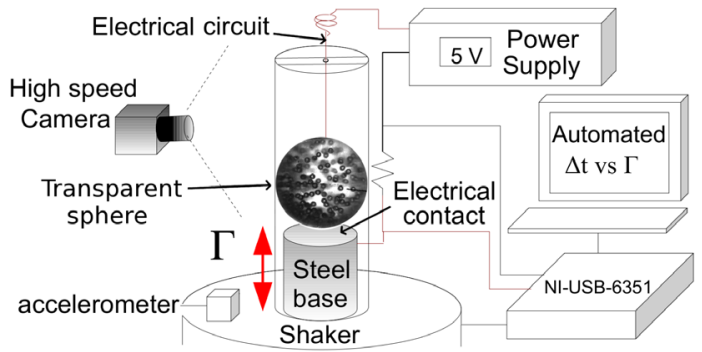

FIG. 1 (color online). A sphere partially filled with grains is placed on a vibrating plate. The bouncing time is measured by electrical contact and registered as a function of $\Gamma$. A high-speed video is taken to visualize the particle dynamics inside the sphere. 
(0.08 $\mathrm{mm}$ diameter). The wire was connected to one terminal of a power supply (dc, $5 \mathrm{~V}$ ) and the plate to the other one. A DAQ device NI-USB-6351 was used to capture the peaks in the voltage signal generated when the sphere touched the plate. $\Delta t$ was determined from the time interval between two consecutive peaks and registered as a function of $\Gamma$. The resulting bifurcation diagram (BD) allowed us to find the conditions at which steady modes occurred. These modes were filmed at $1000 \mathrm{fps}$ with a IDTN3 camera (see movie in the Supplemental Material [26]). The videos also allowed us to ensure that the circuit did not affect the BB dynamics in comparison with the one described by an unwired sphere.

The above process was carried out at $f=14 \mathrm{~Hz}=1 / T$ for the three configurations described in Figs. 2(a)-2(c). The empty sphere exhibits a periodic orbit $P_{1}^{2}$ in the interval $1<\Gamma<2$, see Fig. 2(a). This notation means that the ball motion has a period of 2 vibration cycles and describes one kind of trajectory during the orbit [27]. Out of this region, a chaotic dynamics prevails. On the other hand, the liquidfilled sphere displays a $P_{1}^{1}$ orbit at $\Gamma \sim 1(\Delta t / T=1)$ that turns into a $P_{2}^{2}$ orbit in the range $1.2 \leq \Gamma \leq 1.6$, see Fig. 2(b). In these modes, a liquid jet synchronized with the vibration rises inside the ball, see snapshots in Fig. 2(d). This removes rebound energy and reduces the flight time of the ball $[28,29]$. The synchronization is lost when $\Gamma>1.6$, and the irregular liquid splash generates a chaotic behavior. Contrastingly, the ball partially filled with grains bounces mainly in steady orbits $P_{1}^{n}$, with $n=1,2,3 \ldots$, see Fig. 2(c). Even a small mass of particles, $m=1 \mathrm{~g}\left(V_{f}=2.6 \%\right)$ almost stabilizes the ball in three fixed points (indicated by $\left.P_{1}^{1}, P_{1}^{2}, P_{1}^{3}\right)$. For $5.5 \mathrm{~g}<m<10 \mathrm{~g}\left(V_{f} \sim 15 \%-30 \%\right)$, the sphere rebounds in a steady $P_{1}^{1}$ orbit in a broad range of $\Gamma$ (notably, when $m \simeq 7.5 \mathrm{~g}$ ). Under such conditions, the impact excites the grains, and there is enough room to form a confined granular gas; the gas collapses with the following impact, and this process of "excitation and collapse" is repeated continuously, see Fig. 2(e). For $V_{f} \simeq 50 \%$, a bifurcation replaces the $P_{1}^{1}$ orbit. Finally, when $V_{f}=100 \%$, the ball "becomes solid" and describes a fully chaotic dynamics.

Let us focus on the stable $P_{1}^{1}$ mode described by the grainfilled sphere. Figure 3 shows the BB dynamics for different values of $\Gamma$ (the case $m=7.5 \mathrm{~g}$ is taken as an example). Note that there is a shift in the impact phase $(\varphi)$ that grows linearly with $\Gamma$ and also that the impact occurs during the ascent phase $(-\pi / 2<\varphi<\pi / 2)$, see Figs. 3(a), 3(b). For $1<\Gamma \leq 3.8$, the trajectories in Fig. 3(a) show that the sphere describes a tiny rebound after which it sticks on the plate (sticking zone), until the condition $\Gamma \sin \phi>1$ is satisfied
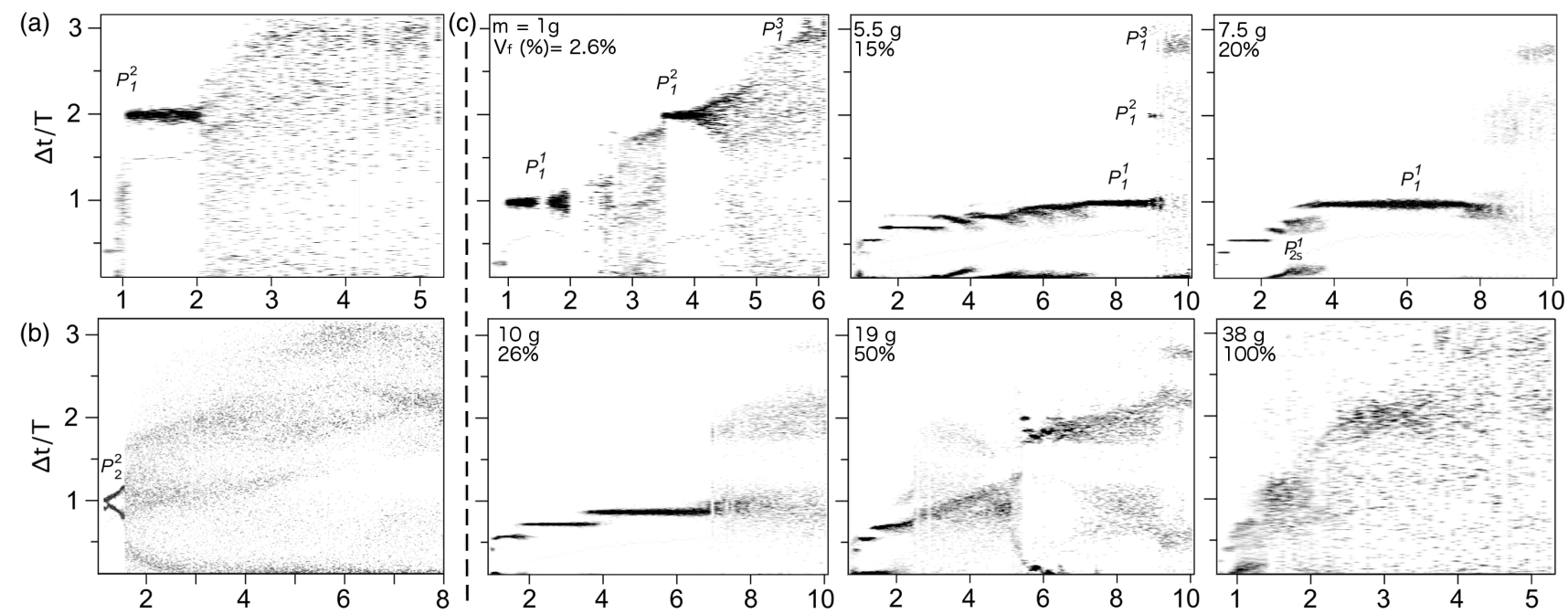

(d) Liquid (oil -300 cSt) $\quad P_{2}^{2}(\Gamma=1.0-1.6)$

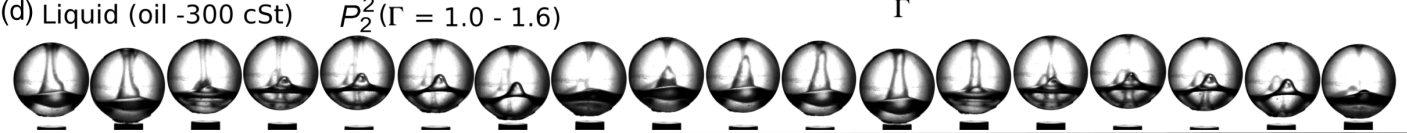

(e) Grains $(2 \mathrm{~mm}) \quad P_{1}^{1}(\Gamma=3.5-8.0)$
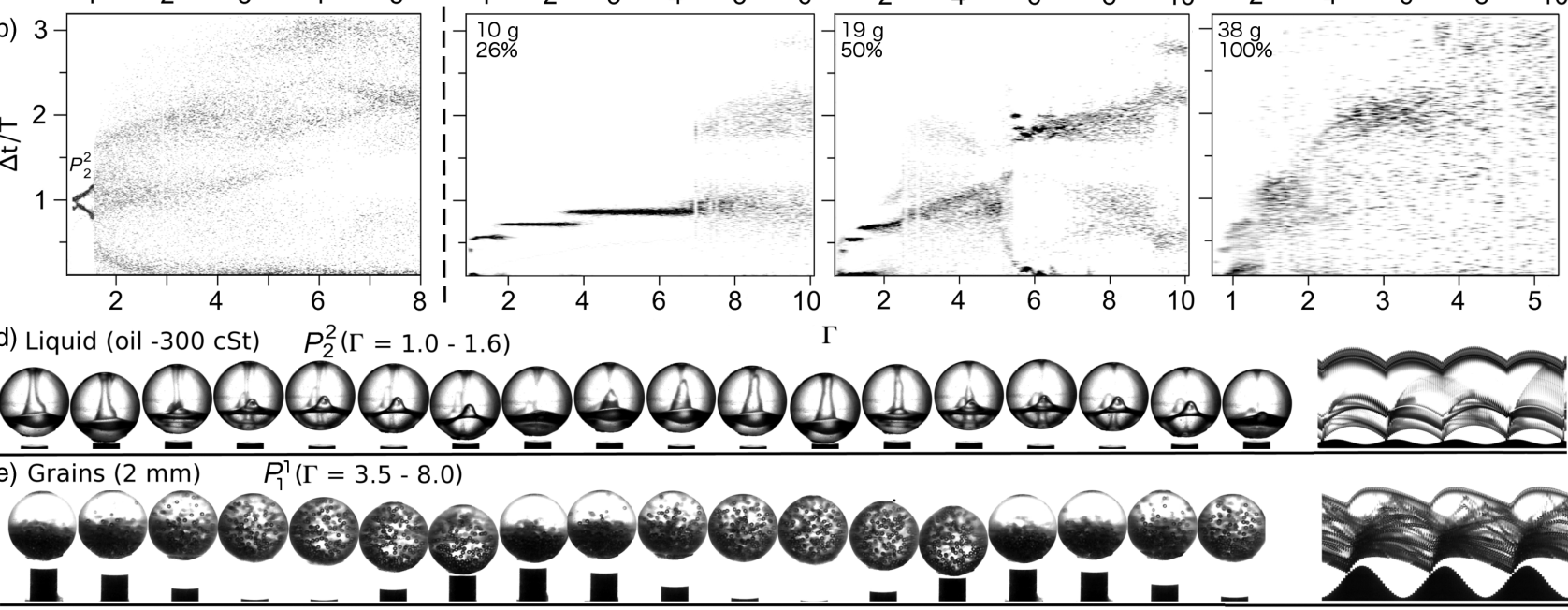

$\Gamma$
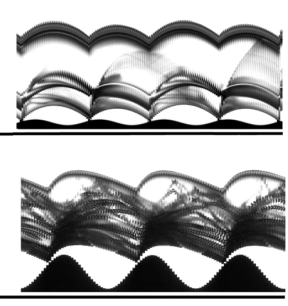

FIG. 2. BDs of a spherical container: (a) empty, (b) with $7.5 \mathrm{~g}$ of oil (300 cSt), and (c) with different masses $m$ of $2 \pm 0.2 \mathrm{~mm}$ glass beads ( $V_{f}$ is the ratio of the volume occupied by the grains to the cavity volume). The BDs are reproducible if they are obtained by increasing or reducing $\Gamma$; a slight hysteresis can appear, but the main features remain unaltered. [(d), (e)] Snapshots taken each $0.01 \mathrm{~s}$ and space-time pictures illustrating periodic orbits of a sphere with $7.5 \mathrm{~g}$ of (d) liquid, and (e) grains. Note: In (d) we used oil to visualize the jet formation. The dynamics was considerably more chaotic with less viscous fluids (in particular, with water). 

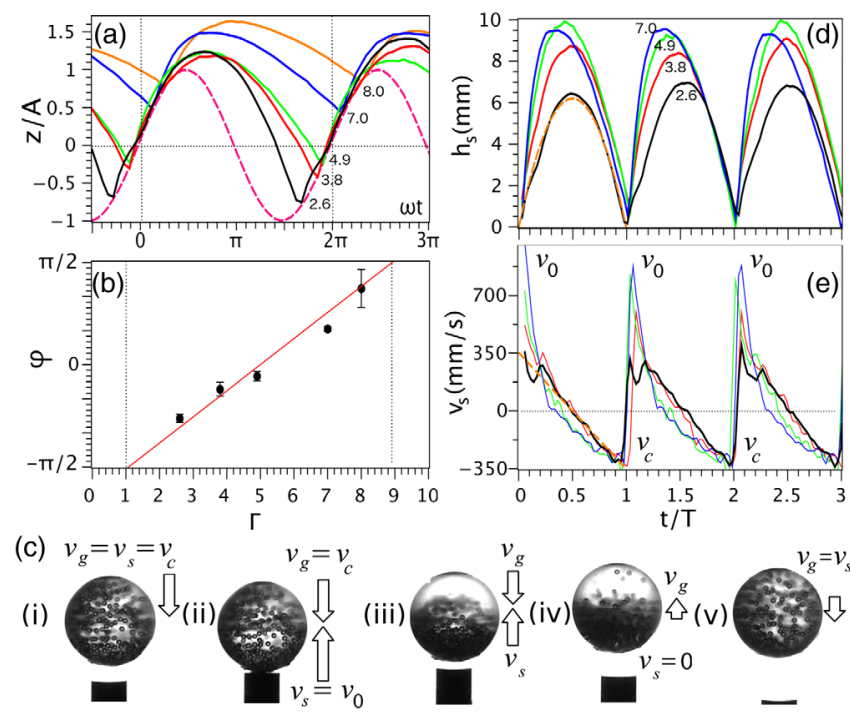

FIG. 3 (color online). Stationary bouncing dynamics of a grainfilled sphere for $V_{f}=20 \%$ ( $P_{1}^{1}$ orbits): (a) Sphere position $z$ normalized by $A$, as a function of $\omega t$ for $\Gamma=2.6$ (black), 3.8 (red), 4.9 (green), 7.0 (blue), and 8.0 (orange). The dashed line corresponds to the sinusoidal motion of the plate. (b) Impact phase $\varphi$ as a function of $\Gamma$. (c) Snapshots showing the grainssphere interaction during a typical impact in the $P_{1}^{1}$ state (see text). (d) $h_{s}$ and (e) $v_{s}$ vs $t / T$, see text.

$[14,30]$; then, the sphere takes off again and describes a longer second flight. These two bounces of $\Delta t / T<1$ are indicated by $P_{2 s}^{1}$ in Fig. 2(c) ("s" alludes to sticking). Then, the periodic motion could be explained considering the sticking zone as a reset mechanism that erases the past history of the system [30]; however, $P_{1}^{1}(\Delta t / T=1)$ remains even out of this zone $(\Gamma>3.8)$, where a different mechanism seems to govern [Fig. 3(c)]. (i) Before the collision, the granular gas falls with an average velocity $v_{g}$ equal to the sphere velocity $v_{s}$. (ii) During the impact, the ball is practically free of grains (which are still falling). Thus, it suffers a partially elastic collision and leaves the plate with $v_{s}=v_{0}$. (iii) The sphere exchanges momentum with the grains through multiple collisions during its parabolic ascent. (iv) This exchange removes energy from the sphere, which is dramatically decelerated. (v) Then, the energy transferred mostly to the grains generates a granular gas, and the process starts again.

Figures 3(d) and 3(e) show the flying height of the sphere $h_{s}$ measured from the impact with the plate $\left(h_{s}=0\right)$ and its velocity $v_{s}=d h_{s} / d t$ as a function of $t / T$. These plots reveal that $v_{0}$ increases as $\Gamma$ does, but the main feature is that, independently of $\Gamma$, the speed before the collision is always the same $\left(v_{c} \sim 350 \mathrm{~mm} / \mathrm{s}\right)$. Interestingly, this is the impact speed of a solid ball in a $P_{1}^{1}$ orbit for $f=T^{-1}=$ $14 \mathrm{~Hz}$ (orange dashed parabola). Let us remind that a solid ball describes this orbit if the platform compensates the energy loss from the collision so that the ball lands and departs at the same speed $v_{i}$ with a parabolic flight time $T$
[1,5]. Thus, $v_{i}=g T / 2=350 \mathrm{~mm} / \mathrm{s}$. For a partially elastic solid ball [13,14], $v_{i}=v_{0}$ is satisfied only in a short range of $\Gamma$. In our case, the system self-regulates: it does not matter for $v_{0}$; the grains dissipate the energy, and the phase is shifted, such that $v_{c}=g T / 2$ is satisfied over a wide range. Since the injected energy must be dissipated by interparticle collisions, the self-regulation mechanism depends on the number of particles and filling fraction. Therefore, if the grains are very few or if there is not enough space to form the gas, the dissipation is less efficient and the steady state disappears. This explains the chaotic dynamics for small and large filling fractions in Fig. 2(c).

Another important factor in the BB dynamics must be the grain size. We explored this parameter using glass beads of different diameters in Figs. 4(a)-4(c) $(m=7.5 \mathrm{~g}$ in all cases). Note that the steady state previously observed for $d=2 \mathrm{~mm}$ disappears. With larger grains $d=4 \mathrm{~mm}$, this happens because the number of particles (collisions) is considerably reduced from $\sim 700$ to 86 beads, and the dissipation does not compensate for the injected energy. In fact, a larger mass of $4 \mathrm{~mm}$ particles is needed to reach the "dissipation capacity" observed with $2 \mathrm{~mm}$ beads, see Fig. 4(j). For smaller particles $(d \sim 300 \mu \mathrm{m})$, collective motions of grains [see snapshots in Figs. 4(d)-4(g)] increase friction, which becomes the most efficient dissipation mechanism. High-speed videos show that the tiny grains remain at rest within the $\mathrm{BB}$ at $\Gamma \sim 1$, small surface perturbations appear as $\Gamma$ increases, and they evolve to oscillons or waves depending on $f$. Oscillons appeared at $f=14 \mathrm{~Hz}$ in the interval $2.8<\Gamma<3.2$. These structures consist of a cavity formed by the grains during one period and a jet projected upwards due to the cavity collapse during the second period, see Figs. 4(c) and 4(d). Note that the ball describes $P_{1}^{1}$ while the grains are in $P_{1}^{2}$. If the frequency is reduced to $10 \mathrm{~Hz}$, the oscillons are replaced by alternating waves in the same interval [Fig. 4(g)]. At $f=14 \mathrm{~Hz}$, waves appear combined with jets as asymmetric oscillons in $3.2<\Gamma<3.6$ [Fig. 4(e)]. This combination induces a broadening with $\Gamma$ of the $P_{1}^{1}$ noise up to switch to $P_{1}^{2}$ at $\Gamma \sim 5.4$ [Fig. 4(f)]. For $\Gamma>5.5$, the synchronized motion disappears. A phase diagram compiling the above observations is shown in Fig. 4(h).

Similar patterns were observed with grains [31] and liquid [32] inside spherical containers fixed to a sinusoidal oscillation. Then, the geometry seems important for the emergence of oscillons and waves depending on the frequency. We propose that the resulting pattern is related to the distance the grains can travel inside the sphere during one cycle. According to Figs. 4(d) and 4(i), the central jet travels a distance $l_{o}=D_{c}-s$, where $s$ can be obtained from the snapshots or from the volume of the spherical cap occupied by the grains: $V_{g}=m / \phi \rho=\pi s^{2}\left(\frac{3}{2} D_{c}-s\right) / 3$, with $\phi \sim 0.64$. For $m=7.5 \mathrm{~g}$, one finds $s \approx 1.0 \pm 0.1 \mathrm{~cm}$. Since the oscillon rises under gravity, its flight time is $t=\sqrt{2 l_{o} / g} \approx 0.074 \mathrm{~s}$, which corresponds to $f_{o}=T^{-1}=13.5 \mathrm{~Hz}$. On the other 

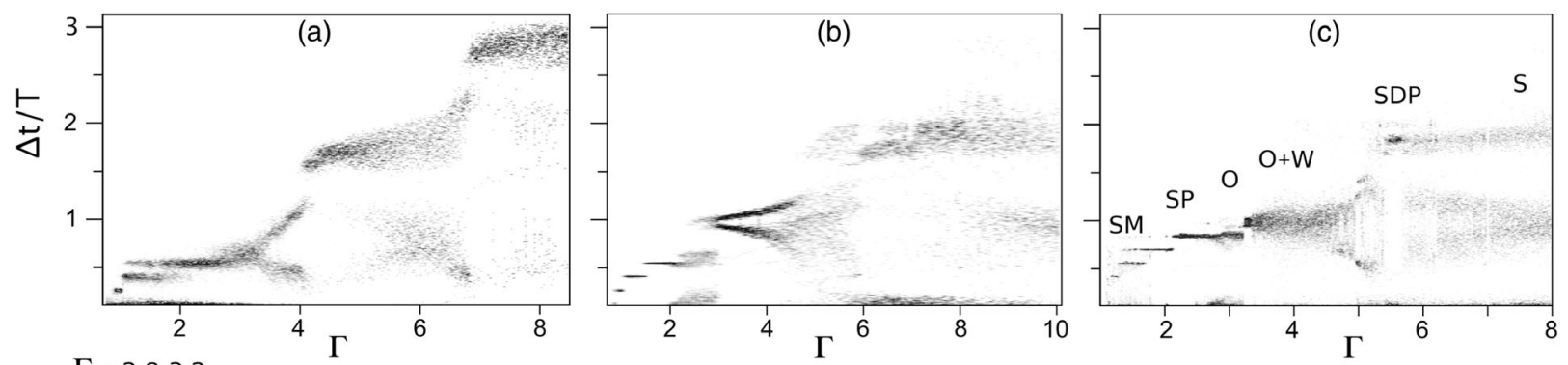

(d)
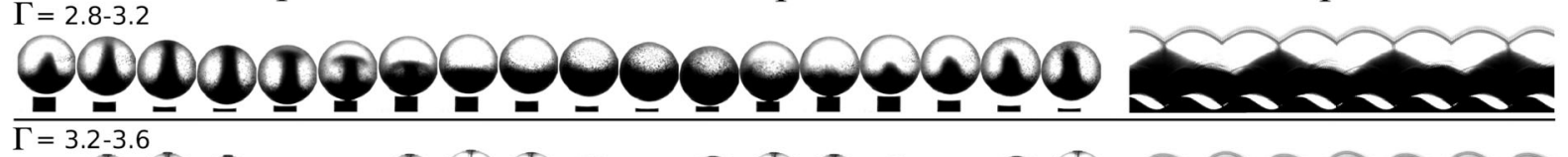

(e)

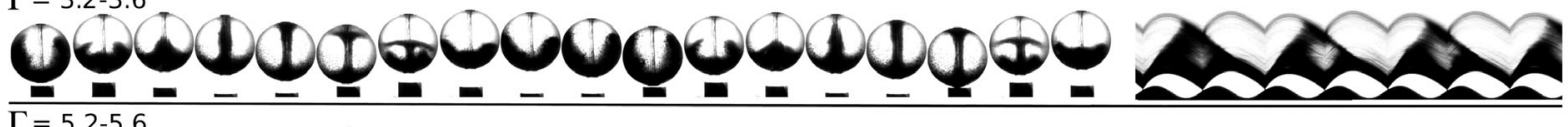

(f)

$\Gamma=5.2-5.6$

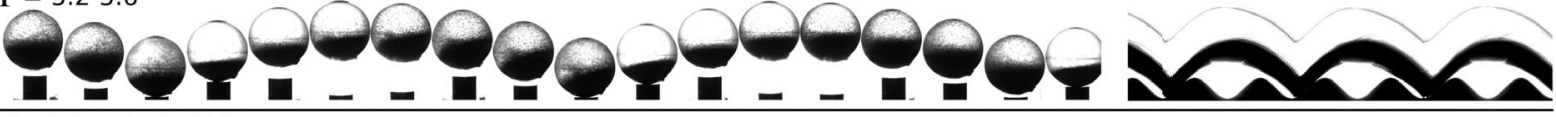

$\Gamma=2.6-3.6(10 \mathrm{~Hz})$

(g)
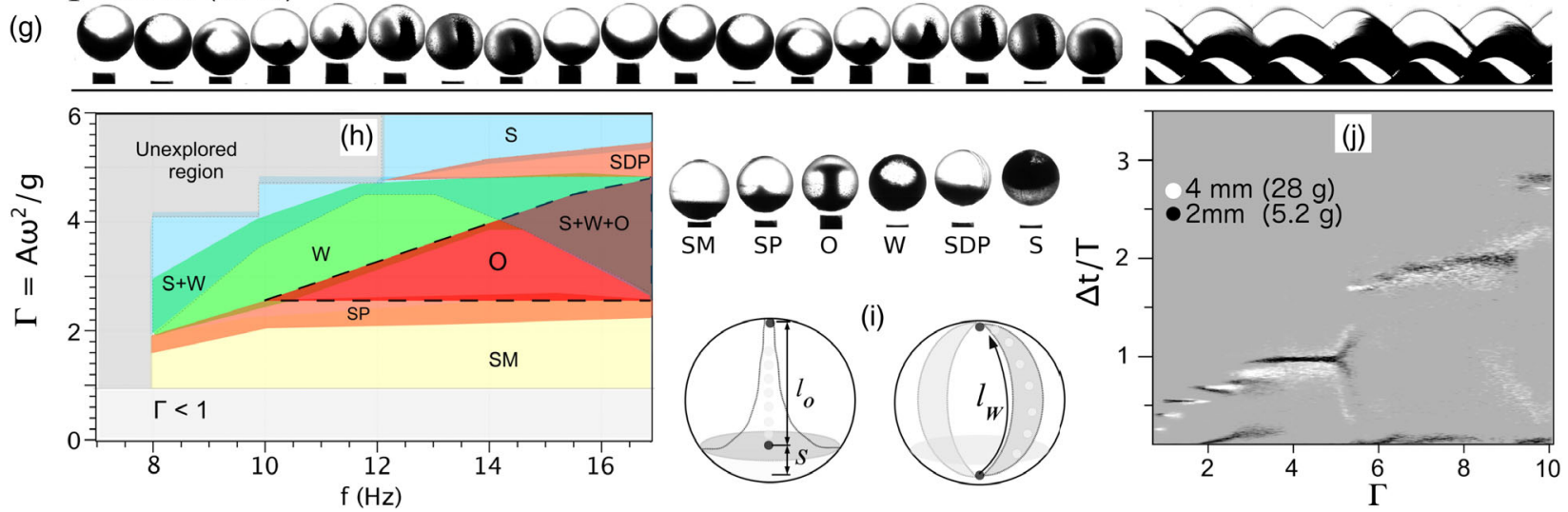

FIG. 4 (color online). BDs displayed by a sphere partially filled with glass particles of different diameters: (a) $4 \pm 0.05 \mathrm{~mm}$, (b) $1.0 \pm 0.2 \mathrm{~mm}$, and (c) $\sim 300 \mu \mathrm{m}$. In all cases, $m=7.5 \mathrm{~g}$. For the smaller particles, a rich variety of vibrating modes was observed: (d) stable oscillons, (e) asymmetric oscillons, (f) doubling period orbits, and (g) waves (the corresponding parametric regions are indicated in (c). (h) Phase diagram summarizing the observed orbits: static material (SM), surface perturbations (SP), oscillons (O), waves (W), static double period (SDP), and sloshing (S). Oscillons only appear in the interval $12 \mathrm{~Hz} \lesssim f \lesssim 16 \mathrm{~Hz}$. (i) Particles trajectories for oscillons and waves. (j) Similar BDs for different grain size indicate similar dissipation capacity.

hand, the wave travels over the container walls a larger distance $l_{w} \sim \pi D_{c} / 2$. Thus, $t \approx 0.109 \mathrm{~s}$, which corresponds to a lower frequency $f_{w} \sim 9 \mathrm{~Hz}$. Therefore, our geometrical analysis predicts the right frequencies in accordance with the phase diagram. Regarding the $P_{1}^{2}$ orbit at $\Gamma \sim 5.4$, it was deduced theoretically in Ref. [7], and it is independent on the bouncing geometry. Is worth mentioning that static charge and air drag over the small particles [29] could influence their dynamics, as it was found in other granular systems [33,34]. In addition, the ball stiffness can also be relevant. These effects will be addressed in a future work.

Summarizing: a grain-filled ball on a vibrating plate displays a rich variety of periodic orbits and synchronized patterns depending on the dissipation mechanism (frictional or collisional). A worthy highlight is a period-1 orbit in the collisional regime produced by the formation and collapse of a granular gas into the cavity during one cycle, which is possible only for small filling volumes and large number of particles. How the system self-regulates to maintain this stationary state independently of the vibration conditions is an open question to be tackled theoretically or through simulations.

F. P.-V., F. L., and S. D. thank to CONACyT, Morecar T-REX project, and F. R. S.-FNRS, respectively, for financial support. F. P.-V. was a beneficiary of the Belspo-Marie Curie Program at the University of Liège.

*fpacheco@ifuap.buap.mx

[1] J. J. Barroso, M. V. Carneiro, and E. E. N. Macau, Phys. Rev. E 79, 026206 (2009).

[2] E. Fermi, Phys. Rev. 75, 1169 (1949). 
[3] D. Sternad, M. Duarte, H. Katsumata, and S. Schaal, Phys. Rev. E 63, 011902 (2000).

[4] N. A. Burnham, A. J. Kulik, G. Gremaud, and G. A. D. Briggs, Phys. Rev. Lett. 74, 5092 (1995).

[5] N. B. Tufillaro, T. M. Mello, Y. M. Choi, and A. M. Albano, J. Phys. (Paris) 47, 1477 (1986).

[6] S. Dorbolo, D. Volfson, L. Tsimring, and A. Kudrolli, Phys. Rev. Lett. 95, 044101 (2005).

[7] S. Dorbolo, F. Ludewig, and N. Vandewalle, New J. Phys. 11, 033016 (2009).

[8] Y. Couder, S. Protiere, E. Fort, and A. Boudaoud, Nature (London) 437, 208 (2005).

[9] S. T. Dembiński, A. J. Makowski, and P. Peplowski, Phys. Rev. Lett. 70, 1093 (1993).

[10] C. Becco, N. Vandewalle, J. Delcourt, and P. Poncin, Physica (Amsterdam) 367A, 487 (2006).

[11] H. Chaté, F. Ginelli, G. Grégoire, F. Peruani, and F. Raynaud, Eur. Phys. J. B 64, 451 (2008).

[12] A. Kudrolli, G. Lumay, D. Volfson, and L. S. Tsimring, Phys. Rev. Lett. 100, 058001 (2008).

[13] N. B. Tufillaro and A. M. Albano, Am. J. Phys. 54, 939 (1986).

[14] A. Mehta and J. M. Luck, Phys. Rev. Lett. 65, 393 (1990); J. M. Luck and A. Mehta, Phys. Rev. E 48, 3988 (1993).

[15] S. B. Savage, J. Fluid Mech. 194, 457 (1988).

[16] S. Douady, S. Fauve, and C. Laroche, Europhys. Lett. 8, 621 (1989).

[17] H. K. Pak and R. P. Behringer, Phys. Rev. Lett. 71, 1832 (1993).

[18] F. Melo, P. B. Umbanhowar, and H. L. Swinney, Phys. Rev. Lett. 75, 3838 (1995).

[19] P. B. Umbanhowar, F. Melo, and H. L. Swinney, Nature (London) 382, 793 (1996).

[20] C. Salueña, T. Poschel, and S. E. Esipov, Phys. Rev. E 59, 4422 (1999).
[21] X.-M. Bai, L. M. Keer, Q. Jane Wang, and R. Q. Snurr, Granular Matter 11, 417 (2009).

[22] M. N. Bannerman, J. E. Kollmer, A. Sack, M. Heckel, P. Mueller, and T. Pöschel, Phys. Rev. E 84, 011301 (2011).

[23] M. Sánchez, G. Rosenthal, and L. A. Pugnaloni, J. Sound Vib. 331, 4389 (2012).

[24] A. Sack, M. Heckel, J. E. Kollmer, F. Zimber, and T. Poschel, Phys. Rev. Lett. 111, 018001 (2013).

[25] F. Pacheco-Vázquez and S. Dorbolo, Sci. Rep. 3, 2158 (2013).

[26] See Supplemental Material at http://link.aps.org/ supplemental/10.1103/PhysRevLett.113.118001. The movie shows different orbits described by a grain-filled ball bouncing on a vibrating plate: a period-1 orbit independent of the vibration conditions in the collisional regime, and periodic patterns such as oscillons and waves observed in the frictional regime.

[27] R. Leine and T. F. Heimsch, Physica (Amsterdam) 241D, 2029 (2012).

[28] T. W. Killian, R. A. Klaus, and T. T. Truscott, Phys. Fluids 24, 122106 (2012).

[29] These absolute effects must be differentiated from the relative ones (related to the grains and ball size) that determine the number of collisions and contact surface.

[30] T. Gilet, N. Vandewalle, and S. Dorbolo, Phys. Rev. E 79, 055201(R) (2009).

[31] H. M. Jaeger, J. B. Knight, C.-h. Liu, and S. R. Nagel, MRS Bull. 19, 25 (1994).

[32] J. W. Miles and D. M. Henderson, Annu. Rev. Fluid Mech. 22, 143 (1990).

[33] M. E. Mobiüs, B. E. Lauderdale, S. R. Nagel, and H. M. Jaeger, Nature (London) 414, 270 (2001); M. E. Mobiüs, X. Cheng, G. S. Karczmar, S. R. Nagel, and H. M. Jaeger, Phys. Rev. Lett. 93, 198001 (2004); Phys. Rev. E 72, 011304 (2005).

[34] N. Burtally et al., Science 295, 1877 (2002). 